\title{
On the impact of the pilot density in the channel estimation of MC-CDMA systems
}

\author{
Carlos Ribeiro, ${ }^{\#, * 1}$, Atílio Gameiro ${ }^{\# 2}$ \\ ${ }^{*}$ Instituto de Telecomunicações / Universidade de Aveiro \\ Campo Universitário, 3810 - 193 Aveiro, Portugal \\ ${ }^{1}$ cribeiro@av.it.pt \\ ªmg@det.ua.pt \\ *Instituto Politécnico de Leiria \\ Morro do Lena, Alto Vieiro, 2411 - 901 Leiria, Portugal
}

\begin{abstract}
In this paper we investigate the impact of the pilot density (and associated pilot distances for both frequency and time domains) in the performance of the channel estimation for MC-CDMA systems, when using dedicated sub-carriers to transmit pilots with 2-D rectangular patterns.

The analytical formulation of the channel estimation MSE for the single-user downlink scenario with a generic channel estimation scheme for a given power efficiency is presented.

The attained results go beyond the commonly accepted rule of thumb that suggests that an oversampling factor of two will result in a good trade-off between performance and pilot overhead.

The analytical formulation was validated by system simulations. A scenario using the 2-D LS-DFT channel estimation scheme confirmed the analytical result. The lowest channel estimation MSE for a given value of power efficiency is achieved when the pilot density is the one that closely fulfils the 2-D sampling theorem.
\end{abstract}

\section{INTRODUCTION}

Multicarrier Code Division Multiple Access (MC-CDMA) [1] is a strong candidate to be the transmission support for the $4^{\text {th }}$ generation broadband wireless applications. Extraction of accurate channel state information is crucial to achieve high spectral efficiency, with emphasis on demodulation/decoding and resource allocation operations. Blind estimation techniques that need to gather a large amount of information to perform the estimation exhibit a poor performance in mobile systems where the channel varies rapidly under the influence of Doppler's effect and multipath propagation. To achieve better performance pilot-aided channel estimation techniques are commonly preferred, with emphasis on twodimensional (2-D) filtering algorithms and associated 2-D pilot patterns [2]-[4]. Detailed studies of optimum pilot pattern for 2-D channel estimation schemes for both uplink and downlink scenarios are present in [5],[6].

To the authors knowledge there is no in-depth analytical study on the influence of the pilot distances (frequency and time) and density on the performance of the channel estimator. This issue was addressed in [3], were a rule of thumb suggested that an oversampling factor of two would result in a good trade-off between performance and pilot overhead. In this paper this analytical study is introduced for the singleuser downlink scenario. The feasibility of the analytical model is substantiated by system simulations using a 2-D Least Squares - discrete Fourier transform (LS-DFT) channel estimation scheme and BRAN-A broadband wireless channel model [7].

\section{SYSTEM MODEL}

Let us consider MC-CDMA modulation over $N c$ subcarriers for transmission over a multipath Rayleigh fading wireless channel, with a discrete-time channel impulse response (CIR) given by,

$$
\tilde{h}[n]=\sum_{l=0}^{L-1} \alpha_{l} \delta\left[n-\tau_{l}\right]
$$

where $L$ is the number of channel paths, $\alpha_{l}$ is the complex value of path $l$ and $\tau_{l}$ is the delay of path $l$. The paths are assumed to be statistically independent, with normalized average power, $\sum_{l=0}^{L-1} \sigma_{l}^{2}=1$, where $\sigma_{l}^{2}$ is the average power of path $l$. The time dependence of the CIR is not present in the notation for simplicity.

We assume that the CIR remains constant for the duration of the symbol and that the insertion of a long enough cyclic prefix $(\mathrm{CP})$ in the transmitter assures that the orthogonality of the sub-carriers in maintained after transmission.

The system's frame structure is made-up of Ns symbols, each carrying $N c M$-ary PSK or QAM modulated subcarriers. To assist in the channel estimation process, dedicated sub-carriers with-in the frame convey pilots in a 2-D rectangular pattern with pilot separation in frequency and time domains of, respectively, $N f$ and $N t$.

The 2-D rectangular pilot pattern, like any regular 2-D pattern [8]-[9], corresponds to a non-singular matrix $\mathbf{V}$ represented by 2 basis vectors $\mathbf{v}_{i}=\left[\begin{array}{ll}N t & 0\end{array}\right]^{T}$ and $\mathbf{v}_{n}=\left[\begin{array}{ll}0 & N f\end{array}\right]^{T}$,

$$
\mathbf{V}=\left[\mathbf{v}_{i}: \mathbf{v}_{n}\right]=\left[\begin{array}{cc}
N t & 0 \\
0 & N f
\end{array}\right]
$$


such that the set of the pattern points is defined by,

$$
\left\{\mathbf{y}=[n, i]^{T}: \mathbf{y}=\mathbf{V} \mathbf{x}, \forall \mathbf{x} \in \mathbb{Z}^{2}\right\},
$$

and the pilot pattern density $D$ is $D=|\operatorname{det}(\mathbf{V})|^{-1}=(N t N f)^{-1}$.

Suppose that the receiver is perfectly synchronized and is disturbed by independent and identically (iid) zero mean Additive White Gaussian noise (AWGN) with a variance $\sigma_{N}^{2}$.

\section{MSE ANALYTICAL FORMULATION}

The receiver's channel estimator starts by obtaining the LS estimates $\mathbf{H}_{L S}$ of the channel for the set of pilot positions with-in the frame $\mathrm{P}$,

$$
H_{L S}[n, i]=H[n, i]+\frac{N[n, i]}{S[n, i]}, \forall(n, i) \in \mathrm{P}
$$

where $\mathbf{H}, \mathbf{N}$ and $\mathbf{S}$ are $N c \times N s$ matrices that contain, respectively, the channel's frequency response (CFR), the noise samples and the transmitted symbols and after uses a 2-D DFT interpolator to obtain the estimates in the remaining frame positions.

Interpreting the LS estimates as noisy samples of the CFR, for the interpolator filter to be able to recover the CFR without aliasing, the pilot distances $N f$ and $N t$ should fulfill the 2-D sampling theorem, with maximum frequencies dependent of the channel's minimum coherence time and minimum coherence bandwidth, that are, respectively, functions of the mobile speed and channel's maximum propagation delay $\tau_{\text {max }}$ [2],

$$
\begin{aligned}
& N f \leq\left(\tau_{\max } \Delta f\right)^{-1}, \\
& N t \leq\left(f_{D} T_{S}\right)^{-1}
\end{aligned}
$$

where $\Delta f$ is the sub-carrier separation, $f_{D}$ is the Doppler frequency and $T_{S}$ is the MC-CDMA symbol duration including CP.

In the absence of noise, the discrete CFR can be reconstructed using an ideal 2-D sinc filter, $w_{P}[n, i]$,

$$
w_{p}[n, i]=\operatorname{sinc}\left[\frac{1}{N f} n\right] \operatorname{sinc}\left[\frac{1}{N t} i\right],
$$

that oversamples the LS estimates by a factor $N f$ in the frequency domain and $N t$ in the time domain,

$$
H[n, i]=\sum_{k=-\infty}^{+\infty} \sum_{l=-\infty}^{+\infty} H_{S}[n+k, i+l] w_{P}[k, l],
$$

where,

$$
H_{S}[n, i]=\left\{\begin{array}{l}
H[n, i],\{n, i\} \in \mathrm{P} \\
0, \text { remaining }
\end{array} .\right.
$$

The real LS estimates are noisy samples of the channel, that are used as the input to the 2-D estimation filter $w[n, i]$, to obtain the channel estimate $\hat{H}[n, i]$,

$$
\hat{H}[n, i]=\sum_{k=-\infty}^{+\infty} \sum_{l=-\infty}^{+\infty} H_{L S}^{\prime}[n+k, i+l] w[k, l],
$$

where,

$$
H_{L S}^{\prime}[n, i]=\left\{\begin{array}{l}
H_{L S}[n, i],\{n, i\} \in \mathrm{P} \\
0, \text { remaining }
\end{array} .\right.
$$

Splitting $\mathbf{H}_{\mathrm{LS}}^{\prime}$ in $\mathbf{H}_{\mathrm{LS}}^{\prime}=\mathbf{H}_{\mathrm{S}}+\mathbf{N}_{\mathrm{S}}$, where the elements of the matrix containing the noise samples $\mathbf{N}_{\mathrm{s}}$ are defined by,

$$
N_{S}[n, i]=\left\{\begin{array}{l}
\frac{N[n, i]}{S[n, i]},\{n, i\} \in \mathrm{P} \\
0, \text { remaining }
\end{array},\right.
$$

the channel estimation can be rewritten as,

$$
\begin{aligned}
\hat{H}[n, i] & =\sum_{k=-\infty}^{+\infty} \sum_{l=-\infty}^{+\infty}\left(H_{S}[n+k, i+l]+N_{S}[n+k, i+l]\right) w[k, l] \\
& +H[n, i]-\sum_{k=-\infty}^{+\infty} \sum_{l=-\infty}^{+\infty} H_{S}[n+k, i+l] w_{P}[k, l] \\
& =H[n, i]+\sum_{k=-\infty}^{+\infty} \sum_{l=-\infty}^{+\infty} H_{S}[n+k, i+l]\left(w[k, l]-w_{P}[k, l]\right) \\
& +\sum_{k=-\infty}^{+\infty} \sum_{l=-\infty}^{+\infty} N_{S}[n+k, i+l] w[k, l]
\end{aligned}
$$

which can be interpreted as if the channel estimation is the summation of the real channel $H[n, i]$ with two terms that negatively affect the estimate. The first term is due to the imperfect estimation filter,

$$
\sum_{k=-\infty}^{+\infty} \sum_{l=-\infty}^{+\infty} H_{S}[n+k, i+l]\left(w[k, l]-w_{P}[k, l]\right),
$$

and the second is due to the presence of noise, $\sum_{k=-\infty}^{+\infty} \sum_{l=-\infty}^{+\infty} N_{S}[n+k, i+l] w[k, l]$.

Defining the error filter $w_{e}[n, i]=w[n, i]-w_{P}[n, i]$, the estimation error of the $n$-th sub-carrier of the $i$-th symbol can be written as,

$$
\begin{aligned}
e[n, i]= & \hat{H}[n, i]-H[n, i] \\
= & \sum_{k=-\infty}^{+\infty} \sum_{l=-\infty}^{+\infty} H_{S}[n+k, i+l] w_{e}[k, l] \\
& +\sum_{k=-\infty}^{+\infty} \sum_{l=-\infty}^{+\infty} N_{S}[n+k, i+l] w[k, l] \\
& =H_{S}[n, i] * w_{e}[n, i]+N_{S}[n, i] * w[n, i]
\end{aligned} .
$$


Applying 2-D discrete-time Fourier transform (DTFT) to (14), it can be equivalently written as,

$$
E\left(w_{n}, w_{i}\right)=H_{S}\left(w_{n}, w_{i}\right) W_{e}\left(w_{n}, w_{i}\right)+N_{S}\left(w_{n}, w_{i}\right) W\left(w_{n}, w_{i}\right) .
$$

Using the 2-D discrete Parseval's theorem [9], the channel estimation mean square error (MSE) can be written as,

$$
\sigma_{e}^{2}=\frac{1}{4 \pi^{2}} \int_{-\pi}^{\pi} \int_{-\pi}^{\pi}\left|E\left(w_{n}, w_{i}\right)\right|^{2} d w_{n} d w_{i} .
$$

Considering that the noise $N_{S}\left(w_{n}, w_{i}\right)$ and the channel's response $H_{S}\left(w_{n}, w_{i}\right)$ are independent and recalling that $\mathbf{N}_{S}$ is made-up of AWGN samples,

$$
\begin{aligned}
\left|E\left(w_{n}, w_{i}\right)\right|^{2} & =S_{H_{S}}\left(w_{n}, w_{i}\right)\left|W_{e}\left(w_{n}, w_{i}\right)\right|^{2} \\
& +\left|N_{S}\left(w_{n}, w_{i}\right)\right|^{2}\left|W\left(w_{n}, w_{i}\right)\right|^{2}, \\
& =S_{H_{S}}\left(w_{n}, w_{i}\right)\left|W_{e}\left(w_{n}, w_{i}\right)\right|^{2}, \\
& +\sigma_{N_{S}}^{2}\left|W\left(w_{n}, w_{i}\right)\right|^{2}
\end{aligned}
$$

where $S_{H_{S}}\left(w_{n}, w_{i}\right)=\left|H_{S}\left(w_{n}, w_{i}\right)\right|^{2}$. Recalling that $N_{S}[n, i]$ is a sampled version of $N[n, i]$ with a density $D$ and considering that the pilots are transmitted with unit energy, $\sigma_{N_{S}}^{2}=\sigma_{N}^{2} D$. The channel estimation MSE can be rewritten as,

$$
\begin{aligned}
\sigma_{e}^{2} & =\frac{1}{4 \pi^{2}} \int_{-\pi}^{\pi} \int_{-\pi}^{\pi} S_{H_{S}}\left(w_{n}, w_{i}\right)\left|W_{e}\left(w_{n}, w_{i}\right)\right|^{2} d w_{n} d w_{i} \\
& +\frac{\sigma_{N}^{2} D}{4 \pi^{2}} \int_{-\pi}^{\pi} \int_{-\pi}^{\pi}\left|W\left(w_{n}, w_{i}\right)\right|^{2} d w_{n} d w_{i}
\end{aligned}
$$

The sampled channel's power spectral density (PSD) $S_{H_{S}}\left(w_{n}, w_{i}\right)$ is a replicated and scaled version of the original channel's PSD $S_{H}\left(w_{n}, w_{i}\right)$ [8],

$$
S_{H_{S}}\left(w_{n}, w_{i}\right)=\frac{1}{(N t N f)^{2}} \sum_{i=0}^{N t-1} \sum_{n=0}^{N f-1} S_{H}\left(w_{n}-\frac{2 \pi}{N f} n, w_{i}-\frac{2 \pi}{N t} i\right) .
$$

Fig. 1 presents the relationship between $S_{H_{S}}\left(w_{n}, w_{i}\right)$ and $S_{H}\left(w_{n}, w_{i}\right)$, assuming that the channel's PSD is limited in both frequency axes and that the pilot distances $N f$ and $N t$ are small enough to fulfill the 2-D sampling theorem, according to (5). The top figure represents the original channel's PSD and the bottom one the sampled version. The position of the replicas is defined by [8],

$$
\mathbf{U}=[\mathbf{u} 1: \mathbf{u} 2]=\left[\begin{array}{cc}
2 \pi / N t & 0 \\
0 & 2 \pi / N f
\end{array}\right] \text {. }
$$

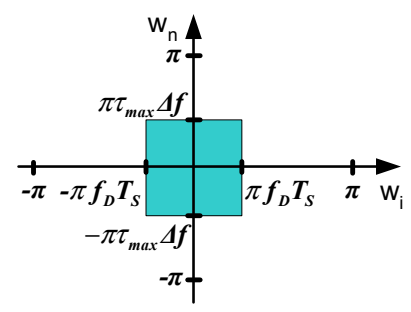

a)

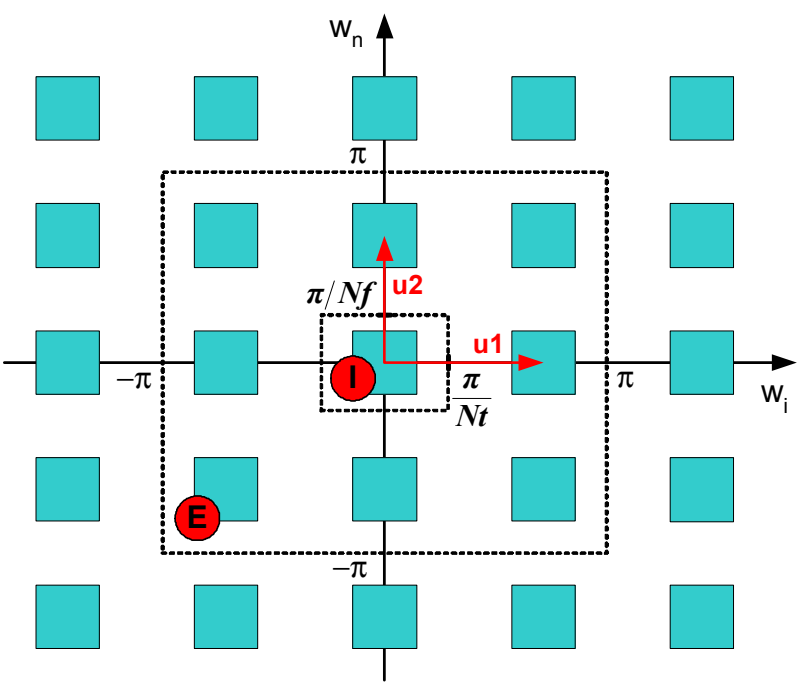

b)

Fig. 1. Channel's PSD: a) original; b) sampled.

Keeping in mind that the 2-D sinc filter is only non-zero in the area I (marked in Fig. 1), the 2-D DTFT of the error filter is given by,

$W_{e}\left(w_{n}, w_{i}\right)=\left\{\begin{array}{l}W\left(w_{n}, w_{i}\right)-N t N f, \quad\left|w_{i}\right| \leq \pi / N t \wedge\left|w_{n}\right| \leq \pi / N f \\ W\left(w_{n}, w_{i}\right), \text { remaining }\end{array}\right.$

Considering that the interpolator filters most of the energy of the channel replicas inside the area E (marked in Fig. 1), the error introduced by the first term of (18) in this area is neglectable and the channel estimation MSE can be rewritten,

$$
\begin{aligned}
\sigma_{e}^{2} & \approx \frac{1}{4 \pi^{2}} \iint_{I} S_{H_{S}}\left(w_{n}, w_{i}\right)\left|W\left(w_{n}, w_{i}\right)-N t N f\right|^{2} d w_{n} d w_{i} \\
& +\frac{\sigma_{N}^{2} D}{4 \pi^{2}} \int_{-\pi}^{\pi} \int_{-\pi}^{\pi}\left|W\left(w_{n}, w_{i}\right)\right|^{2} d w_{n} d w_{i}
\end{aligned}
$$

In the area I $\left(\left|w_{i}\right| \leq \pi / N t \wedge\left|w_{n}\right| \leq \pi / N f\right)$, the sampled channel's PSD is just a scaled version of the original channel's PSD,

$$
S_{H_{S}}\left(w_{n}, w_{i}\right)=\frac{1}{(N t N f)^{2}} S_{H}\left(w_{n}, w_{i}\right)=D^{2} S_{H}\left(w_{n}, w_{i}\right) .
$$

Using (23) in (22), 


$$
\begin{aligned}
\sigma_{e}^{2} & \approx \frac{D^{2}}{4 \pi^{2}} \iint_{I} S_{H}\left(w_{n}, w_{i}\right)\left|W\left(w_{n}, w_{i}\right)-(N t N f)\right|^{2} d w_{n} d w_{i} \\
& +\frac{\sigma_{N}^{2} D}{4 \pi^{2}} \int_{-\pi}^{\pi} \int_{-\pi}^{\pi}\left|W\left(w_{n}, w_{i}\right)\right|^{2} d w_{n} d w_{i} \\
& \approx \frac{D^{2}}{4 \pi^{2}} \iint_{I} S_{H}\left(w_{n}, w_{i}\right)\left|W\left(w_{n}, w_{i}\right)\right|^{2} d w_{n} d w_{i} \\
& -\frac{2 D}{4 \pi^{2}} \iint_{I} S_{H}\left(w_{n}, w_{i}\right) \operatorname{Re}\left\{W\left(w_{n}, w_{i}\right)\right\} d w_{n} d w_{i} \\
& +\frac{D}{4 \pi^{2}} \iint_{I} S_{H}\left(w_{n}, w_{i}\right) d w_{n} d w_{i} \\
& +\frac{\sigma_{N}^{2} D}{4 \pi^{2}} \int_{-\pi}^{\pi} \int_{-\pi}^{\pi}\left|W\left(w_{n}, w_{i}\right)\right|^{2} d w_{n} d w_{i} \\
& \approx A D^{2}+\left(\sigma_{N}^{2} E_{F}-B\right) D+E_{C}
\end{aligned}
$$

where,

$$
\begin{aligned}
& A=\frac{1}{4 \pi^{2}} \iint_{I} S_{H}\left(w_{n}, w_{i}\right)\left|W\left(w_{n}, w_{i}\right)\right|^{2} d w_{n} d w_{i} \\
& B=\frac{2}{4 \pi^{2}} \iint_{I} S_{H}\left(w_{n}, w_{i}\right) \operatorname{Re}\left\{W\left(w_{n}, w_{i}\right)\right\} d w_{n} d w_{i} \\
& E_{C}=\frac{1}{4 \pi^{2}} \iint_{I} S_{H}\left(w_{n}, w_{i}\right) d w_{n} d w_{i} \\
& E_{F}=\frac{1}{4 \pi^{2}} \int_{-\pi}^{\pi} \int_{-\pi}^{\pi}\left|W\left(w_{n}, w_{i}\right)\right|^{2} d w_{n} d w_{i}
\end{aligned}
$$

The MC-CDMA system's energy per bit $E b$ is given by,

$$
E b=\frac{\bar{S}_{\text {symbol }} T_{S}}{b_{\text {data }}}
$$

where $\bar{S}_{\text {symbol }}$ is the average MC-CDMA symbol power and $b_{\text {data }}$ is the number of data bits sent per symbol. Due to the presence of pilot sub-carriers in the frame, the average number of data bits per symbol depends on the pilot density $D$,

$$
b_{\text {data }}=\frac{N c}{S F} \log _{2}(m)(1-D),
$$

where $S F$ is the spreading factor and $m$ is the size of the constellation. Replacing in (26),

$$
E b=\frac{\bar{S}_{\text {symbol }} T_{S} S F}{\log _{2}(m)(1-D) N c} .
$$

The one-sided noise PSD NO is given by,

$$
N 0=\frac{\sigma_{N}^{2}}{N c \Delta f}=\frac{\sigma_{N}^{2} t_{S}}{N c},
$$

where $t_{S}$ is the MC-CDMA symbol duration (without CP). The system's power efficiency $E b / N 0$ is defined by,

$$
E b / N 0=\frac{\bar{S}_{\text {symbol }}}{\sigma_{N}^{2}} \frac{T_{S}}{t_{S}} \frac{S F}{\log _{2}(m)(1-D)} .
$$

Expressing the noise power as a function of the power efficiency,

$$
\sigma_{N}^{2}=(E b / N 0)^{-1} \bar{S}_{\text {symbol }} \frac{T_{S}}{t_{S}} \frac{S F}{\log _{2}(m)(1-D)},
$$

the channel estimation MSE can be directly expressed as a function of $E b / N_{0}$, by replacing in (24),

$$
\sigma_{e}^{2} \approx A D^{2}+\left(\frac{(E b / N 0)^{-1} \bar{S}_{\text {symbol }} S F}{\log _{2}(m)(1-D)} \frac{T_{S}}{t_{S}} E_{F}-B\right) D+E_{C}
$$

Equation (32) shows that the channel estimation MSE is dependent of the CFR, the noise present in the process and the frequency response of the estimation scheme used.

\section{A. 2-D LS-DFT channel estimator}

The 2-D LS-DFT channel estimation scheme uses the 2-D sinc filter expressed in (6) to perform the interpolation that will return the estimates of the channel for the frame positions where data sub-carriers were transmitted. In this scenario, $w=w_{p}$, the error filter $w_{e}=0$ and (15) is reduced to

$$
E\left(w_{n}, w_{i}\right)=N_{S}\left(w_{n}, w_{i}\right) W\left(w_{n}, w_{i}\right),
$$

as the interpolator does not cause any distortion. Examining (22) it is straightforward that the channel estimation MSE with this scheme is $\sigma_{e}^{2}=\sigma_{N}^{2}$, or expressed in terms of power efficiency by rewriting (32),

$$
\sigma_{e}^{2}=(E b / N 0)^{-1} \bar{S}_{\text {symbol }} \frac{T_{S}}{t_{S}} \frac{S F}{\log _{2}(m)} \frac{1}{(1-D)}
$$

A closer examination of (34) puts in evidence that, for a given value of power efficiency, the lowest MSE achieved with this channel estimation scheme is for the lowest value of pilot density $D$ (with associated pilot distances $N f$ and $N t$ ) that closely fulfill the 2-D sampling theorem.

\section{SimULATION RESULTS}

A simulation scenario was implemented where $N c=1024$ sub-carriers were QPSK modulated. The system has a carrier frequency $f_{c}=5 \mathrm{GHz}$ and a sampling interval $\Delta t=10 \mathrm{~ns}$. The transmission of symbols was carried out over BRAN-A model channels [7]. The surfaces presented in Fig. 2 summarize the 
attained results. The red surfaces represent the simulation results while the analytical values are represented by the blue ones. The green dot points out the best density $D$ (and respective pair $(N t, N f))$. Surfaces in the first row were obtained for a MT speed of $10 \mathrm{~km} / \mathrm{h}$, while in the second row the speed was set to $200 \mathrm{~km} / \mathrm{h}$. Each column represents a different power efficiency value, $E b / N 0$. From left to right, $10 d B, 20 d B, 30 d B$ and $40 d B$.

Observing the surfaces, we can conclude that in the area of interest, where the 2-D sampling theorem is fulfilled (there is no aliasing and the channel estimator performs the best), the analytical formulation of the channel estimation MSE closely follows the simulations results. This validates the approximation performed in the formulation process. Outside this area the surfaces diverge, as it would be expected, since the analytical formulation does not take into account the distortion caused by the aliasing. The optimum pilot density attained by simulation confirms the analytical result.

\section{CONCLUSIONS}

The analytical formulation of the channel estimation MSE as a function of the pilot density (and distances) for a given power efficiency is presented.

Simulation results validate the analytical formulation in the area of interest, confirming that in the simulated scenario the optimum density is the one that closely fulfils the 2-D sampling theorem.

The channel estimation MSE is very sensitive to the fulfillment of the sampling frequency in the time axis, due to
Doppler spectrum energy being concentrated near the Doppler frequency. Even a small overlap of the channel PSD replicas causes a considerable increase of the channel estimation MSE.

\section{ACKNOWLEDGMENT}

This work was supported by the CELTIC project "WISQUAS" (CP2-035).

\section{REFERENCES}

[1] N. Yee, J.-P. Linnarz, and G. Fettweis, "Multi-carrier CDMA in indoor wireless radio networks", in Proc. PIMRC'93, pp. 109-113, Yokohama, Japan, Sept. 1993.

[2] P. Hoeher, S. Kaiser, and P. Robertson, "Two-dimensional pilotsymbol-aided channel estimation by Wiener filtering," in Proc. ICASSP'97, pp. 1845-1848, Munich, Germany, April 1997.

[3] S. Kaiser, and P. Hoeher, "Performance of multi-carrier CDMA systems with channel estimation in two dimensions," in Proc. PIMRC'97, pp. 115-119, Helsinki, Finland, September 1997.

[4] Y. Li, "Pilot-symbol-aided channel estimation for OFDM in wireless systems," IEEE Transactions on Vehicular Technology, Vol. 49, Issue 4, pp.1207-1215, July 2000.

[5] F. Bader, S. Zazo, and J. M. Páez Borrallo, "Optimum pilot pattern for the Uplink Multicarrier-CDMA systems," in Proc. Symp. on Wireless Personal Multimedia Comms., Amsterdam, Netherlands, 1999.

[6] J.-W. Choi, and Y.-H. Lee, "Optimum pilot pattern for channel estimation in OFDM systems," IEEE Transactions on Wireless Communications, Vol. 4, Issue 5, pp. 2083-2088, Sept. 2005.

[7] ETSI Project Broadband Radio Access Networks (BRAN), HIPERLAN Type 2, Technical specification; Physical layer, October 1999.

[8] D. E. Dudgeon, R. M. Mersereau, Multidimensional Digital Signal Processing, Prentice-Hall, New Jersey, 1984.

[9] R. M. Mersereau, T. C. Speake, "The processing of periodically sampled multidimensional signals," IEEE Trans. Signal Processing, vol. ASSP-31, pp. 188-194, Feb. 1983.
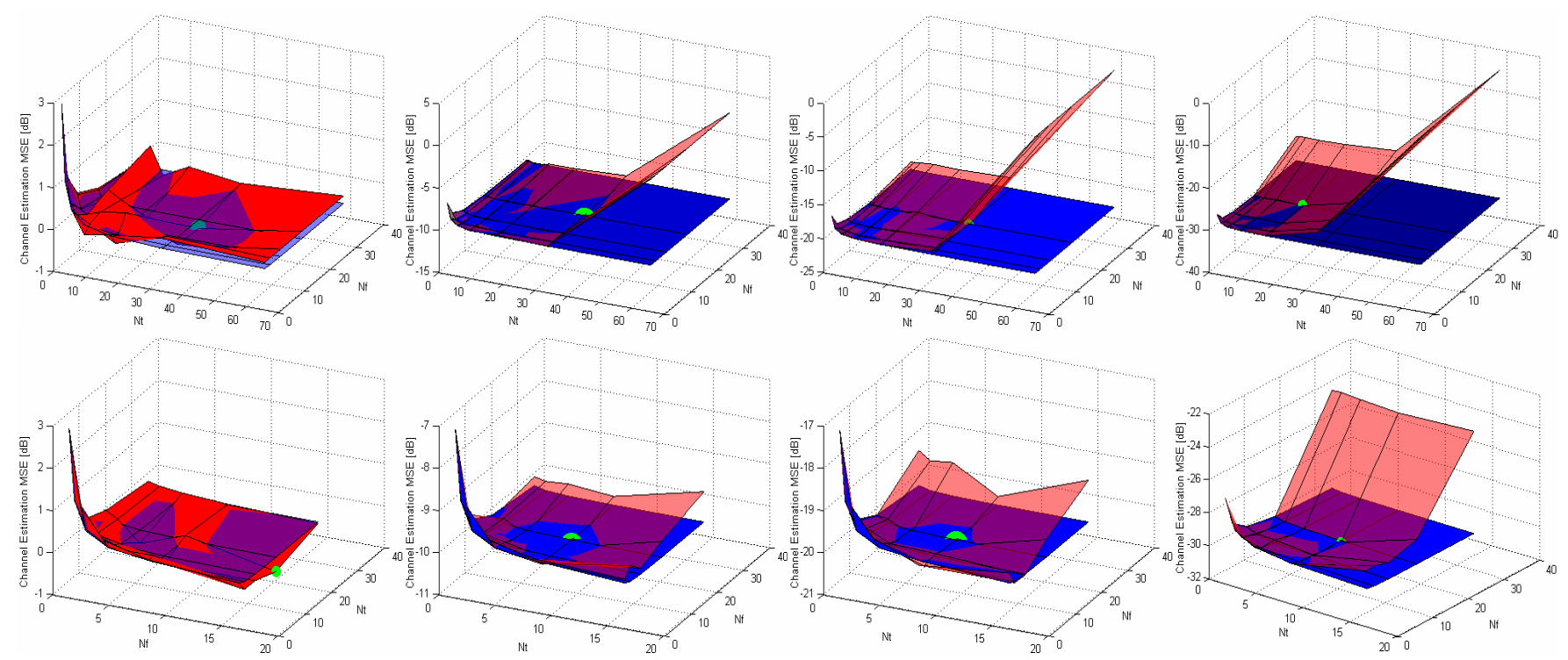

Fig. 2. Channel estimation MSE surfaces 